\title{
Research on Graduates Employability Development from the Perspective of Employers
}

\author{
Zhi-qiong Guo ${ }^{1}$, Bing Bai $^{2}$ \\ ${ }^{1)}$ Business School, Jiangsu Normal University, Xuzhou, Jiangsu, China \\ ${ }^{2)}$ Business School, Jiangsu Normal University, Xuzhou, Jiangsu, China
}

\begin{abstract}
Through the investigation on the part of employers and graduates, this paper analyzes the standards and requirements of employers and the actual situation of graduates employment, and points out that the information asymmetry situation still exists between graduates and employers. The self-orientation of graduates and the needs of the employers exist differences, and there is a certain deviation between the cultivation mode of college students and the demand of the society. It is successful to enhance graduates employability and promote graduates employment, only when the employability of graduates and the talent demands of employers should be consistent and strive to dynamic match.
\end{abstract}

Keywords - college students, employability, Employers

\section{基于用人单位的高校毕业生就业能力提升研究 \\ 郭志琼 白冰 \\ 江苏师范大学商学院, 徐州, 江苏, 中国}

摘 要 本文通过对部分用人单位和毕业生的调查, 分析了用人单位人才需求的标准和要求, 以及毕业生就业的实际情况, 指出毕 业生和用人单位之间的信息不对称的情况依然存在。毕业生自我定位和用人单位需求存在偏差, 大学生培养模式和社会需求存在一定 偏差。只有毕业生能力应当与用人单位的需求达到动态匹配，才能提升毕业生就业能力，进而促进毕业生成功就业。

关键词 大学生, 就业能力, 用人单位

\section{1. 引言}

近年来我国高校毕业生人数一直呈递增状态, 2014 届 毕业生规模达 727 万人, 而 2015 年 2015 届高校毕业生人 数将达 749 万, 就业面临更加严峻的挑战。就业是民生之 本, 经济全球化进程和技术进步在推动经济增长和增加就 业机会的同时, 也必然带来就业的不稳定性和就业者的失 业风险。大学生就业能力与用人单位选才标准无法达到动 态匹配, 导致大学生 “就业难” 与用人单位 “招人难” 矛 盾的产生。大学生培养质量是否与社会需求相匹配, 能否 满足用人单位的要求是高校普遍面临的一个重要课题。

在激烈的就业竞争中, 大学生成功就业要依赖自身的专业 素养和综合素质, 但由于毕业生就业招聘中用人单位对毕 业生的考察往往受到时间、精力等所限, 只能在很短时间
内做出人员招聘的选择, 所以在相当大的程度上毕业生能 否应聘成功取决于在应聘过程中的表现是否可以和用人单 位的需求相匹配。

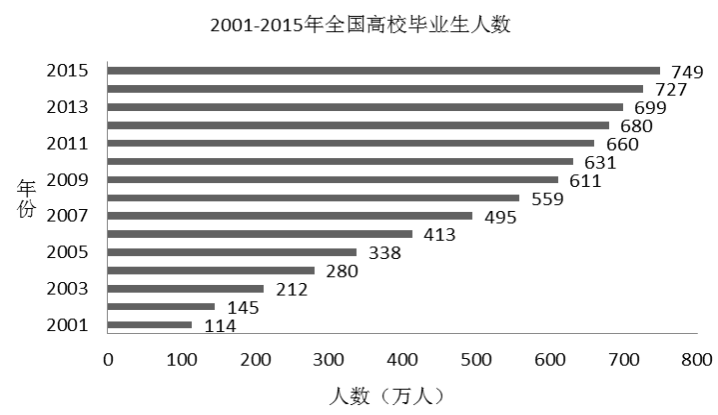

图 1 2001-2015 年全国高校毕业生人数

国家社会科学基金教育学项目支持（资助号：CIA130172） 
由于大学生的大学经历是相似的, 存在很强的同质性, 但他们的学习成绩、综合素质却不尽相同。高校对学生的 评价机制与用人单位对毕业生的评价机制是不同的, 在与 用人单位交流过程中经常会听到 “好学生不一定是好员工” 的说法, 用人单位需要的不仅仅是一个学位, 他们更希望 毕业生能表现出扎实丰富的知识结构和出色的个人品质 等。学生时代主要依靠的是智商, 而职场更注重的是情商。 用人单位招聘的目的招聘的是最合适的人, 而不是最优秀 的, 了解用人单位的选才要求, 针对性的培养有利于成功 就业的关键因素, 对于即将走向社会的毕业生而言就显得 尤为迫切, 同时对于未毕业的学生来说也非常重要。

\section{2. 调查实施}

为了解毕业生就业能力的基本情况及其与用人单位需 求的匹配程度, 以为提高高校毕业生就业能力提出合理建 议, 本研究在文献研究的基础上, 2014 年 9 月对江苏省部 分用人单位以及徐州市某高校 2014 届部分毕业生进行了调 查, 采取直接发放调查问卷和网络问卷相结合的方式进行。 同时对部分用人单位和毕业生进行了深度访谈, 以期对大 学生求职能力更深入的了解。

在调查中, 共调研用人单位 40 家, 其中国有企业 10 家，民营/个体企业 22 家，政府机构/科研或其他事业单位 3 家, 中外合资/外资企业 5 家, 共发放调查问卷 200 份, 有 效问卷 195 份。调研徐州市某高校 2014 届毕业生, 发放调 查问卷 300 份, 其中有效问卷 290 份。

\section{3. 调查结果分析}

在调查中发现, 毕业生的准备与用人单位的需求存在 一定差距。用人单位对毕业生的期望与毕业生在招聘过程 中展示出的能力有一定偏差。

\section{1 用人单位人才需求情况分析}

用人单位性质和提供岗位不同, 对高校毕业生的要求 存在较大的差异。就用人单位的问卷数据显示, 用人单位 在选聘毕业生时候最关注的是人际交往能力、所学专业、 其次是沟通协调能力、解决问题的能力、创新能力等。可 以看出, 专业位居用人单位招聘人才标准的第二位, 第一 位和第三位均是人际能力。可以发现国内用人单位与其他 国家选才标准在逐步接近, 反映出经济全球化趋势带来的 人才需求标准的变化。从调查结果可以看出大多数用人单 位非常看重学生的实践能力, 认为实践能力较强的学生动 手能力、创新能力以及人际能力等要好于单纯成绩优秀的 学生, 同时进入职场后实践能力强的学生社会适应要好。

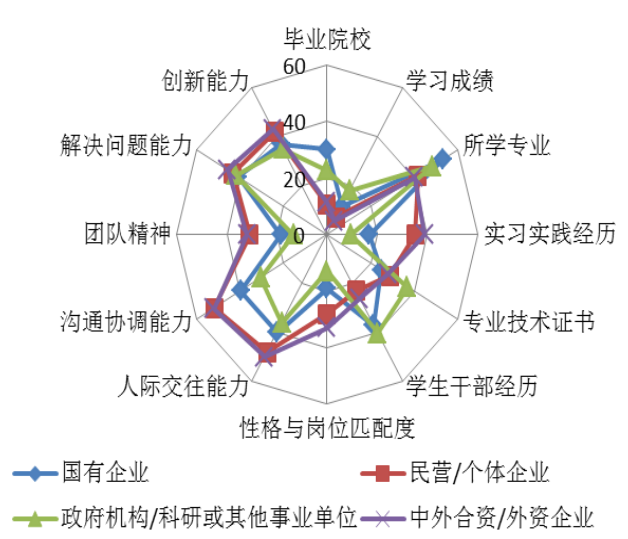

图 2 不同类型用人单位招聘员工标准

建议大学生应该注重自身的全面发展, 提高就业竞争力, 以期与用人单位期望的就业能力相匹配。

从图 2 中可以看出, 国有企业、政府机构和事业单位 等更加重视学习成绩、学生干部经历、毕业院校等背景的 标准, 民营、三资企业更看重的是毕业生的实习实践经历、 毕业生性格和岗位的匹配度、沟通能力等。同时从数据中 可以看出解决问题的能力和创新能力近年来已经愈来愈受 到用人单位的重视, 这与信息化时代的要求相吻合。

用人单位对毕业生的整体满意度达到 $82 \%$, 其中对毕 业生的团队精神、职业道德、专业技能等表示非常满意和 比较满意, 但对毕业生的创新精神和解决问题的能力未达 到 $50 \%$ 用人单位的肯定。说明目前毕业生的整体素质得到 了用人单位肯定, 调查中用人单位对毕业生提出了要具有 扎实的专业基础, 较宽的知识面, 较强的动手能力等要求, 反映很多大学生好高丞远, 期望值过高, 希望他们可以在 大学期间调整好就业心态, 端正态度, 加强自身的综合素 质, 这样才能赢得社会青睐。高校应该加强对学生创新能 力和解决问题能力的培养, 同时毕业生人际能力和动手能 力应受到更多的关注。

参加访谈的用人单位表示, 在招聘过程中对大学生的 学习平时成绩并不是非常看重, 大学生较强的社交能力和 应变能力是吸引用人单位青睐的重要因素, 而这部分同学 往往能够得到用人单位的肯定, 进而获得更好的机会。希 望高校可以将就业服务贯穿到教学环节, 尽早让学生了解 用人单位选拨人才的要求和标准, 使学生不仅仅有较强的 专业背景, 更能满足社会人才需求的期望。

\section{2 毕业生求职情况分析}

对 2014 届毕业生的调查显示, 大多数毕业生已经能够 对就业形势给予重视, 争取就业机会具有较强的主动性。 
您认为在校期间哪些环节对您帮助最大

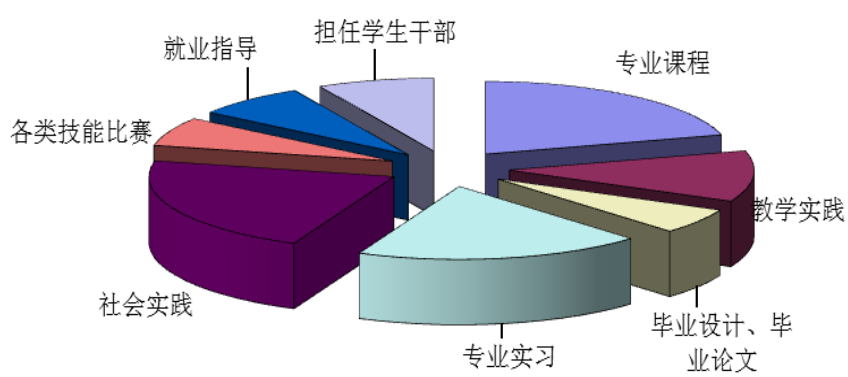

图 3 毕业生对大学期间各教学环节的评价

\section{（1）已就业毕业生调查分析}

已就业的毕业生认为大学期间对自己帮助最大的依次 是: 社会实践、专业技能、专业实习、教学实践、学生干 部经历、就业指导、各类比赛、论文设计等 (见图 3)。最 需要具备的能力依次为: 人际交往能力、沟通表达能力、 专业技能、应变和创新能力、解决问题能力、团队协作能 力、心理调适能力。

已就业毕业生数据显示, 顺利就业最重要的能力是人 际交往能力、沟通表达能力以及问题分析解决的能力, 而 胜任工作最重要的能力有工作态度、知识和技能以及分析 问题处理问题的能力。在招聘环节, 用人单位由于时间精 力所限不可能充分了解每一位毕业生的情况, 那么在有限 的时间内展示出自身与用人单位相匹配的的素质成为毕业 生是否顺利就业的关键。毕业生通过自身就业过程中的感 悟在自己努力提高的同时传递给其他未就业学生, 尤其是 低年级学生, 在一定程度上实现了良性循环。

\section{（2）未就业毕业生的调查}

通过对未就业毕业生谈话结果显示 “没有积极找工 作”, 和 “个人能力不强” 等被大多数毕业生列为待就业原 因, 也反映出学生就业能力的缺失, 如时间管理能力差, 态度不积极, 综合能力不足等。这部分学生往往存在较强 的被动等待心理, 在面对就业时过于被动, 往往浪费很多 很好的就业机会, 如果他们希望成功就业, 必须能够主动 积极应对就业, 将自己的能力与用人单位要求相匹配, 成 功把自身推销给心仪用人单位。作为高校, 如果就业指导 工作能够更早惠及这部分大学生, 让他们在入学开始就持 续接受就业能力的培养和就业意识的熏陶, 引导其积极参 加各种就业指导活动, 端正就业观念, 提升就业能力, 那 么这部分同学必将在就业中更加自信和积极。

大学生在待人接物、处理问题等方面在一定程度上是
欠缺的, 他们对社会的看法往往理想化、片面化, 缺少工 作和社会经验, 而用人单位往往更讲求 “务实”, 大学生在 学校更多体现的是 “智商”, 而在工作中却更注重是的 “情 商”。职场的优胜劣汰的残酷是很多大学生逃避面对的现 实, 但也恰恰是大学生必须要面对接受的, 要成为职场中 的精英, 就必须在踏入社会前开始准备, 这需要在大学阶 段的培养和提升, 利用在校期间积极参加各种实践实习, 提高自身的综合素质。融入社会, 适者生存, 大学生必须 主动适应社会和工作环境, 不能存在消极和害怕, 要正面 面对挑战, 在加强专业学习的同时, 多参加实践实习活动, 提高自身的社会适应能力。

基于调查情况我们得到如下启示:

(1) 毕业生自我定位和用人单位需求存在偏差, 对于 即将走出校园的大学生而言, 人际能力、解决问题能力、 应变能力等都有待培养和提升, 就业能力的欠缺往往导致 用人单位对其青崃的机会的流失。这为大学生今后如何成 功就业提供了思路和建议。

（2）毕业生和用人单位之间的信息存在不对称，信息 不对称也在一定程度上加大了大学生的能力与用人单位需 求达到良性的动态匹配的难度, 这就造成了部分学生就业 难的状况。这为高校今后培养更有就业竞争力的学生提供 了建议。

（3）大学生培养模式和社会需求存在一定偏差, 高校 在大学生能力与用人单位要求达到动态匹配问题上作用未 很好发挥。高校人才培养和就业工作的优化提升必然以市 场为导向, 高校可以在人才培养和就业指导方面进行有效 探索和改革, 建立一套与社会需求相匹配的的人才培养模 式。

\section{3. 总结与建议}

通过以上分析，进行就业工作思路的探索如下：

（1）提高就业指导服务水平。高校应该实施对大学生 的全程化与个性化结合的就业指导服务。一方面, 就业指 导应该贯穿整个大学生的大学生涯, 全程化的指导培养对 于大学生职业定位, 提高各方面能力以及合理进行职业规 划都有及其重要的意义。另一方面, 就业指导服务应更加 多样化和个性化, 大学生性格、知识结构、经历等都不相 同，在其就业过程中所遇问题也多种多样，这就决定了就 业指导必须是个性化的, 这样才能使就业指导工作真正做 到不流于形式，为毕业生提供高效便捷的服务。

(2) 注重大学生实习实践能力的培养。实习经验是提 升大学生就业水平和就业能力的最重要的因素之一。高校 应加强校企合作, 给在读大学生定期参加实习实践的机会, 创造与用人单位接触的机会, 让大学生早日介入就业市场, 
同时可以在毕业前取得一定的工作经验。另一方面, 用人 单位也可以通过实习期间的考核, 提前选拨优秀的人才进 行培养。

(3) 提高大学生的社交能力和应变能力。大部分学生 不能对自己所学知识很好的学以致用, 在学校注重的是学 习态度, 而用人单位更注重结果; 大学阶段更多的是智商 的体现, 职场展示的侧重是一个人的情商。在大学期间应 该多参加课外活动, 注重培养社交和应变的能力, 这样才 能为就业做一定的准备, 更好的适应社会对人才的需求。

(4) 加强大学生创新能力和职业素养。大学生的创新 意识对就业有很大的影响, 有越来越多的用人单位注重大 学生创新能力。在大学阶段应该加强学生在实践中解决问 题的创新思维, 鼓励学生通过参加创新创意创业大赛, 进 而提高他们的创新能力, 培养创新思维。

（5）优化专业设置。随着社会经济的发展, 学校应该 及时调整或增加课程, 及时反映社会对人才需求的变化, 促进人才培养的市场化。大学生知识结构应与社会对人才 的需求处于匹配状态, 这种匹配必然是动态的。

本研究的调查对象有限, 并不能全面反映不同地区不 同类型用人单位的需求和情况, 同时对毕业生调查也没有 区分专业及性别, 这在一定程度上只能反映大学生共性的 一些问题, 而忽略了个性的深入分析, 今后的研究中将扩 大调查范围, 区分度更加明晰的进行深入研究, 以期取得 更有价值的建议。

\section{参考文献(References)}

[1] Ding Xiao-jiong, "Investigation on the employability of graduates from the perspective of employers: a case study of Shanghai", Journal of higher education, vol.34, no.1, pp.42-50, 2013.

[2] Guo Shengzu, Wang Min and Li Rui, "Reform and adjustmeng of education in the structural contradiction of employment", Education Research, no. 5, pp. 12-19, 2013.

[3] Liu Xue-mei, "The influence of college students' value of career success on employment intention," Journal of higher education, vol. 34, no.5, pp. 76-82, 2013.

[4] Zhu Guowei and Huang Jun, "A study on influencing factors of the college student's employability", Education Research, no. 8, pp. 64-68, 2011..

[5] Geng Yin-hang and Liu Li-ming, "On the promotion of college graduate employability based on the demand of the employers", Fujian jiao уи хие yиап хие bao, no.1, pp.15-18, 2013.

[6] Jiang Cheng, Fan Aiai and Zhang Tian, "Study on the matching degree of graduates' employment expectation: taking Beijing for example," Journal of higher education, vol. 35, no.3, pp. 34-39, 2014.

[7] Miguel Angel Santos Rego, "Purposes of the university and employability challenges in higher education", procedia social and behavioral sciences, no.139, pp.16-25, 2014. 